\title{
Tentonin 3 as a baroreceptor mechanosensor: not a stretch
}

\author{
Jianguo G. Gu and Dan E. Berkowitz
}

Department of Anesthesiology and Perioperative Medicine, School of Medicine, University of Alabama at Birmingham, Birmingham, Alabama, USA.

\begin{abstract}
Mechanical stretch of baroreceptors in the wall of the aortic arch and carotid sinus initiates autonomic reflexes to change heart rate and blood pressure for cardiovascular homeostasis. In this issue of the $J C l$, Lu et al. show that tentonin 3 (TTN3), a recently identified stretch-sensitive ion channel, was present at the vagus afferent nerve endings innervating the aortic arch to function as a baroreceptor. This study expands the molecular profiles of baroreceptors and provides new insights into molecular mechanisms underlying the regulation of cardiovascular functions through baroreceptor function.
\end{abstract}

\section{Baroreceptor reflex}

Blood pressure (BP), the major determinant of organ perfusion, is tightly regulated by negative feedback systems to ensure homeostasis in the event of cardiovascular stress. For example, gravity, one of the greatest stressors for the cardiovascular system, would induce profound hypotension and compromised brain perfusion due to blood pooling if the negative feedback systems failed to function. The main negative feedback system controlled by the autonomic nervous system is mediated by baroreceptors and called the baroreceptor reflex. It constricts veins and arteries and increases heart rate (HR), myocardial contractility, and the cardiac output that sustains BP when moving from a supine to an upright posture.

The baroreceptor reflex is initiated by baroreceptor mechanosensors located at afferent nerve endings in the adventitia of carotid sinuses and the aortic arch (Figure 1). Although a rudimentary understanding of this critically important reflex was known in the 19th century, it took the elegant work of the Austro-German physiologist Heinrich Hering to demonstrate that electrical or mechanical stimulation of the carotid sinus provokes a reflex that induces bradycardia and hypotension (1). In parallel, the Belgian physiologist JeanFrançois Heymans and his son Corneille (who won the Nobel Prize for Physiology and Medicine in 1938), using the parabiosis technique in dogs, proposed that hypertensive bradycardia is a reflex mechanism mediated by the vagal nerve (1). The first detailed studies of the innervation of the carotid body were conducted by De Castro, who demonstrated that the "nude" sensory terminals extending and ramifying through the adventitial layer of the artery are such that they can directly sense the changes in pressure and volume in the vessel (1). Since this time, a robust literature and understanding has developed regarding this reflex including the nature of the electrical activity of the afferents, the role of baroreceptor afferents in coordinating CNS structure and function, and the influence of the baroreceptor reflex on cardiovascular effector organs (2).

\section{Molecular substrates of baroreceptors}

Molecular identities of baroreceptors have long been elusive, although earlier stud-

Related Article: p. 3671

Conflict of interest: The authors have declared that no conflict of interest exists.

Copyright: ( 2020 , American Society for Clinical Investigation.

Reference information: / Clin Invest. 2020;130(7):3412-3415. https://doi.org/10.1172/JCI138120.

ies have proposed that epithelial sodium channel (ENaC), acid-sensing ion channel 2 (ASIC2), and transient receptor potential channel 5 (TRPC5) channels are molecular substrates of baroreceptors (3-6). Genetic deletion of these molecules impaired the baroreceptor reflex. However, there has been a lack of evidence in mammalian sensory afferent nerves showing that these channels are sensitive to physiologically relevant mechanical stimuli, which places their role as baroreceptors into question (7). Recently, Piezo channels (Piezo1 and Piezo2) have been identified as putative baroreceptors (8). Piezo1 and Piezo2 channels are the first confirmed mammalian mechanoreceptors that mediate rapidly adapting (RA) inward currents in response to mechanical stimulation (9). Piezo channels are involved in a broad range of physiological processes in sensory cells, endothelial cells, smooth muscle cells, and red blood cells (10). Both Piezo1 and Piezo2 channels are expressed in nodose ganglion (NG) neurons, which are the somas of vagus nerves, and genetic deletion of Piezo channels impairs baroreceptor reflex function, resulting in labile hypertension and increased BP variability (8). These findings have partially, but not fully, satisfied the criteria for Piezo channels to be considered baroreceptor mechanosensors (11).

In order for an ion channel to qualify as a baroreceptor it must meet several criteria (11). (a) The ion channel must show mechanical sensitivity and be able to transduce mechanical stimuli such as membrane stretch into electrical activity and nerve impulses. (b) It must be expressed in the afferent nerve endings that innervate the carotid sinus and aortic arch. (c) Mechanical activation of the ion channel must induce afferent nerve activity that is consistent with known properties of baroreceptors. (d) Ion channels that are pharmacologically blocked, genetically knocked down, or deleted must abolish the baroreceptor reflex. (e) Blocking, knocking down, or deleting the putative receptor in 


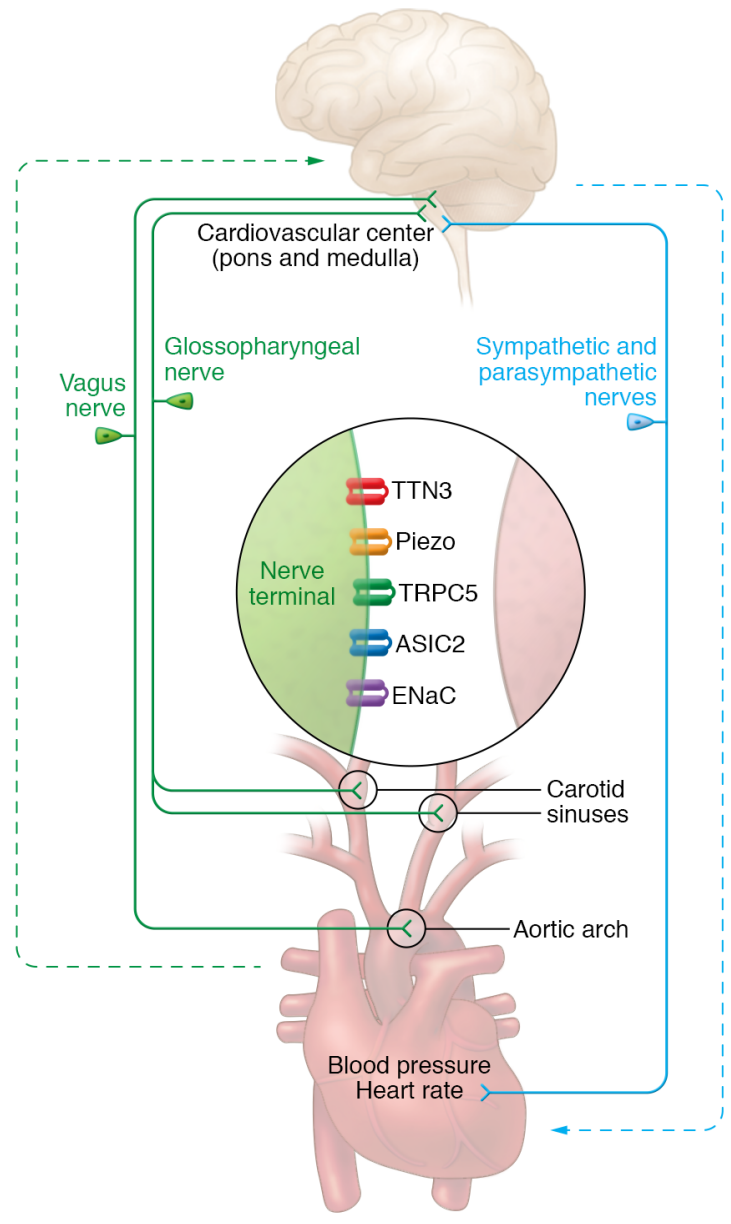

Figure 1. TTN3 is a new baroreceptor in the aortic arch. Diagram illustrating the cellular and molecular elements involved in the baroreceptor reflex. The baroreceptor reflex is initiated by pressureinduced stretch of baroreceptors located at afferent nerve terminals in the aortic arch and carotid sinuses. The vagus nerve and the glossopharyngeal nerve are two afferent nerves that innervate the aortic arch and carotid sinus. Baroreceptor activation generates afferent nerve impulses that are conveyed to the cardiovascular center in the pons and medulla. This subsequently changes autonomic efferent activity, leading to changes in HR and BP. A number of ion channels including ENaC, ASIC2, TRPC5, and Piezo have previously been considered as molecular substrates of baroreceptors. Lu et al. show that TTN3 is a baroreceptor located at the vagus nerve terminals innervating the aortic arch and is essential for the baroreceptor reflex.

awake animals should increase BP lability. Up to now, however, none of the previously identified baroreceptor candidates has fully satisfied these criteria.

\section{TTN3 is a mechanoreceptor}

In this issue of the JCI, Lu et al. have identified tentonin 3 (TTN3) as a baroreceptor in the aortic arch (Figure 1) that well satisfies the criteria described above (12). TTN3 is a mechanoreceptor cloned recently following a bioinformatic investigation. The channel was initially identified in a subpopulation of somatosensory neurons in dorsal root ganglions (DRGs) (13). In both the heterologous expression system and DRG neurons, TTN3 channels mediate slowly adapting (SA) inward currents in response to mechanical stimulation (13). The SA kinetics of TTN3-mediated currents is in sharp contrast to Piezo-mediated inward currents, which are RA in their kinetics (9, 14). In the somatosensory nervous system, TTN3 channels are shown to localize to muscular spindle afferent nerves and are involved in proprioception-related motor coordination (13). Until now, however, it was unknown whether TTN3 was involved in mechanotransduction in other tissues. To explore potential roles of TTN3 as a baroreceptor in the regulation of cardiovascular homeostasis, Lu et al. first characterized TTN3 expression in NG neurons using immunohistochemical methods, because baroreceptors in the aortic arch are vagus nerve endings derived from NGs. The researchers showed that TTN3 immunoreactivity was indeed present in many NG neurons of WT mice but absent in NG neurons of TTN3 gene-knockout mice (Ttn $\left.3^{--}\right)$. To determine whether functional TTN3 channels were present in NG neurons that innervate the aortic arch, the authors retrogradely labeled aortic archprojecting NG neurons using the fluorescent tracer DiI and then applied mechanical pulses and recorded currents from DiI-labeled NG neurons. They showed that mechanical stimulation of aortic arch-projecting NG neurons elicited three types of inward currents: RA currents, intermediately adapting (IA) currents, and SA currents. They further showed that SA currents were absent in DiI-labeled NG neurons of $\operatorname{Ttn}^{-1-}$ mice. Together, these elegant experiments establish TTN3 as a mechanoreceptor responsible for SA currents in aortic arch-projecting NG neurons (12). To satisfy the criteria for TTN3 being a baroreceptor, TTN3 must reside at the afferent nerve terminals in the aortic arch and/or carotid sinus. Lu et al. addressed this key issue by visualizing $\mathrm{TTN}^{+}$nerve terminals in aortic depressor nerves. They used enhanced yellow fluorescent proteinexpressing (EYFP-expressing) transgenic mice along with a tissue-clearing technique to visualize TTN3-expressing nerve fibers and convincingly demonstrated that TTN3 channels were expressed at the vagus afferent terminals in aortic arch adventitia (12). To further determine that TTN3 channels at afferent terminals at the aortic arch sense pressure changes in the aortic lumen, the authors applied hydraulic pressures within the aortic lumen and then recorded from aortic depressor nerves. They found that nerve impulse frequencies were enhanced with increases of aortic pressures in WT mice, but the pressure-induced increases of nerve impulses were substantially abolished in $\operatorname{Ttn} 3^{-1-}$ mice. This finding indicates that TTN3 is required for pressure-evoked impulses in the aortic depressor nerve, providing electrophysiological evidence that activation of TTN3 channels initiates the baroreceptor reflex (12). It is worth noting that pressure-evoked impulses were not completely abolished in the aortic depressor nerves of $T t n 3^{-/-}$mice, which is consistent with the 
idea that multiple mechanoreceptors may function as baroreceptors (Figure 1).

$\mathrm{Lu}$ et al. further provide several lines of evidence to show that TTN3 is a pressure-sensing mechanoreceptor that is essential for the baroreceptor reflex. First, they used a telemetric device to record arterial pressures in freely moving WT and Ttn $3^{--}$mice. Genetic ablation of TTN3 channels resulted in hypertension, tachycardia, and arterial pressure instability (12). These cardiovascular changes were previously seen in animals following the denervation of aortic depressor nerves to abolish the baroreceptor reflex (15). Second, Lu et al. used the designer receptor exclusively activated by designer drug (DREADD) system to show that chemogenetic inhibition or activation of $\mathrm{TTN}^{+} \mathrm{NG}$ neurons resulted in an increase in mean arterial pressures and HRs, or vice versa. This experiment nicely shows the causal relationship between the activity of TTN3-expressing afferent nerves and cardiovascular reflexive responses, strengthening the idea that TTN3 is a baroreceptor (12). Third, to finally confirm that TTN3 is involved in the baroreceptor reflex, $\mathrm{Lu}$ et al. determined whether introducing TTN3 channels back into the NG neurons of $\mathrm{Ttn}^{-/-}$mice would rescue the impaired baroreceptor reflex in these mice. They achieved this goal by delivering TTN3 genes via an adeno-associated virus (AAV) tool into NG neurons of Ttn $3^{-/}$mice. Although Ttn $3^{--}$mice had an impaired baroreceptor reflex, as manifested by their abnormal BPs and HRs, the cardiovascular dysfunctions were normalized following gene delivery of TTN3 channels (12). Taken together, these series of experiments clearly established TTN3 as a baroreceptor in the aortic arch of mice (Figure 1).

\section{Conclusions and future directions}

The findings of Lu et al. identify a role for TTN3 in regulating cardiovascular homeostasis through the baroreceptor reflex. These findings also raise a number of new and important questions. Are TTN3 and previously identified baroreceptors including ASIC2, ENaC, TRPC5, and Piezo channels $(4-6,8)$ redundant mechanosensors for the baroreceptor reflex, or do they each have a distinctive role in the baroreceptor reflex? First, will the roles of TTN3 and Piezo channels, the two types of mechanoreceptors with distinctive current kinetics, differ temporally in the baroreceptor reflex? Second, will the putative baroreceptors identified so far, including TTN3, substantially differ in mechanical sensitivity, such that they function at different ranges of BPs in the baroreceptor reflex? In the somatosensory system, TTN3 channels sense mechanical stretch (13), whereas Piezo2 channels can sense gentle touches $(14,16,17)$, suggesting that the two ion channels may have different mechanical sensitivity. A direct characterization of mechanical sensitivity of each putative baroreceptor in the aortic arch and carotid sinus will help to resolve their range of sensitivity for BPs (both the gain and the set point of the reflex). Interestingly, the baroreceptor reflex set point is such that the gain of the $\mathrm{HR}$ response is greatest in response to a reduction in BP (less so in response to a rise induced using phenylephrine in experiments conducted by the authors) (18). This makes the most sense evolutionarily, given that hypotension and impending loss of blood flow to the brain are much more catastrophic than an increase in BP. As such, the baroreceptor gain (change in HR or R-R interval/change in $\mathrm{BP}$ ) for a given reduction in $\mathrm{BP}$ (induced by hemorrhage or the vasodilator sodium nitroprusside) would be extremely telling about the physiologic role of this and other putative baroreceptor mechanosensors. Third, might putative baroreceptors show differential expression in different types of afferent nerves that innervate the aortic arch and carotid sinus? The aortic arch and carotid sinus are innervated by both $\mathrm{A}$ fibers and $C$ fibers, afferent nerves shown to have different mechanical thresholds (19) and different intrinsic electrophysiological properties (19). Knowing how these putative baroreceptors are expressed in different types of afferent nerves may help us to better understand how they are functionally integrated in the baroreceptor reflex. It is also worth contemplating how polymorphisms or mutations in these mechanosensing genes might contribute to unidentified causes of baroreceptor dysfunction resulting in either hypertension or orthostatic intolerance (20).

Collectively, the identification of TTN3 as a baroreceptor by $\mathrm{Lu}$ et al. (12) highlights the significant role of this mechanosensor in cardiovascular functions, provides insights into the complexity of molecular mechanisms underlying the baroreceptor reflex, and calls for more detailed studies on how different types of baroreceptors may work in a temporally and spatially synergistic manner to control cardiovascular homeostasis.

\section{Acknowledgments}

JGG is supported by the Pain Research Program of the Department of Anesthesiology and Perioperative Medicine at the University of Alabama at Birmingham and by NIH grants DE018661 and DE023090.

Address correspondence to: Dan E. Berkowitz, Department of Anesthesiology and Perioperative Medicine, University of Alabama at Birmingham, 619 South 19th Street, Jefferson Tower, Room 804, Birmingham, Alabama 35249, USA. Phone:205.934.6007; Email:dberkowitz@uabmc.edu.

1. de Castro F. Towards the sensory nature of the carotid body: hering, de castro and heymansdagger. Front Neuroanat. 2009;3:23.

2. Kirchheim HR. Systemic arterial baroreceptor reflexes. Physiol Rev. 1976;56(1):100-177.

3. Abboud FM, Benson CJ. ASICs and cardiovascular homeostasis. Neuropharmacology. 2015;94:87-98.

4. Lu Y, et al. The ion channel ASIC2 is required for baroreceptor and autonomic control of the circulation. Neuron. 2009;64(6):885-897.

5. Drummond HA, Price MP, Welsh MJ, Abboud FM. A molecular component of the arterial baroreceptor mechanotransducer. Neuron. 1998;21(6):1435-1441.

6. Lau OC, et al. TRPC5 channels participate in pressure-sensing in aortic baroreceptors. Nat Commun. 2016;7:11947.

7. Thakore P, Brain SD, Beech DJ. Correspondence: challenging a proposed role for TRPC5 in aortic baroreceptor pressure-sensing. Nat Commun. 2018;9(1):1245.

8. Zeng WZ, et al. PIEZOs mediate neuronal sensing of blood pressure and the baroreceptor reflex. Science. 2018;362(6413):464-467.

9. Coste B, et al. Piezo1 and Piezo2 are essential components of distinct mechanically activated cation channels. Science. 2010;330(6000):55-60.

10. Murthy SE, Dubin AE, Patapoutian A. Piezos thrive under pressure: mechanically activated ion channels in health and disease. Nat Rev Mol Cell Biol. 2017;18(12):771-783.

11. Stocker SD, Sved AF, Andresen MC. Missing pieces of the Piezo1/Piezo2 baroreceptor hypothesis: an autonomic perspective. J Neurophysiol. 2019;122(3):1207-1212.

12. Huan-Jun L, et al. Tentonin 3/TMEM150C senses blood pressure changes in the aortic arch. J Clin Invest. 2020;130(7):3671-3683.

13. Hong GS, et al. Tentonin 3/TMEM150c confers distinct mechanosensitive currents in 
dorsal-root ganglion neurons with proprioceptive function. Neuron. 2016;91(1):107-118.

14. Ikeda R, Cha M, Ling J, Jia Z, Coyle D, Gu JG. Merkel cells transduce and encode tactile stimuli to drive $\mathrm{A} \beta$-afferent impulses. Cell. 2014;157(3):664-675.

15. Thrasher TN. Baroreceptors, baroreceptor unloading, and the long-term control of blood pressure. Am J Physiol Regul Integr Comp Physiol.
2005;288(4):R819-R827.

16. Ranade SS, et al. Piezo2 is the major transducer of mechanical forces for touch sensation in mice. Nature. 2014;516(7529):121-125.

17. Maksimovic S, et al. Epidermal Merkel cells are mechanosensory cells that tune mammalian touch receptors. Nature. 2014;509(7502):617-621.

18. Patton DJ, Triedman JK, Perrott MH, Vidian AA, Saul JP. Baroreflex gain: characterization using autoregressive moving average analysis. Am J Physiol. 1996;270(4 Pt 2):H1240-H1249.

19. Seagard JL, van Brederode JF, Dean C, Hopp FA, Gallenberg LA, Kampine JP. Firing characteristics of single-fiber carotid sinus baroreceptors. Circ Res. 1990;66(6):1499-1509.

20. Kaufmann H, Norcliffe-Kaufmann L, Palma JA. Baroreflex dysfunction. Reply. N Engl J Med. 2020;382(16):1578. 